Artículo científico

Volumen 30(2):333-351. Mayo-agosto, 2019

e-ISSN 2215-3608, doi:1015517/am.v30i2.32905

https://revistas.ucr.ac.cr/index.php/agromeso/index

\title{
Percepciones de cambio climático y respuestas adaptativas de caficultores costarricenses de pequeña escala ${ }^{1}$
}

\section{Climate change perceptions and adaptive responses of small-scale coffee farmers in Costa Rica}

\author{
Bárbara Viguera ${ }^{2}$,Francisco Alpízar ${ }^{2}$,Celia A. Harvey ${ }^{3}$, M. Ruth Martínez-Rodríguez ${ }^{3,4}$, \\ Milagro Saborío-Rodríguez
}

1 Recepción: 17 de abril, 2018. Aceptación: 1 de noviembre, 2018. Este trabajo formó parte del proyecto Adaptación basada en Ecosistemas para pequeños productores de subsistencia y café en América Central (CASCADA) de la Iniciativa Internacional por el Clima (ICI), financiada por el Ministerio Federal de Medio Ambiente, Protección de la Naturaleza, Obras Públicas y Seguridad Nuclear (BMUB) del Gobierno de la República de Alemania.

2 Centro Agronómico Tropical de Investigación y Enseñanza (CATIE). Apdo. 7170, Turrialba, Costa Rica. bviguera@ catie.ac.cr (autor para correspondencia; https://orcid.org/0000-0002-5950-5113); falpizar@catie.ac.cr

3 Conservation International, Moore Center for Science, 2011 Crystal Drive, Suite 500, Arlington, VA, USA 22202. celiaharvey@stanfordalumni.org

4 Programa de las Naciones Unidas para el Ambiente (PNUMA), Unidad de Cambio Climático, Oficina Regional para América Latina y el Caribe, Panamá. ruth.martinez@un.org

5 Universidad de Costa Rica, Escuela de Economía e Instituto de Investigaciones en Ciencias Económicas. Apdo 11501, San Pedro de Montes de Oca, Costa Rica. milagro.saborio@ucr.ac.cr

\section{Resumen}

Introducción. El cambio climático afectará la distribución, productividad y rentabilidad del cultivo de café en América Central, perjudicando las economías nacionales y los medios de vida de los pequeños productores. Es necesario entender cómo el cambio climático está afectando a los pequeños caficultores en la región para promover medidas que permitan enfrentar estos cambios y adaptarse a ellos. Objetivo. El objetivo de este trabajo fue describir los sistemas cafetaleros costarricenses de pequeña escala en dos zonas productivas y explorar los esfuerzos de adaptación al cambio climático que realizan los productores. Materiales y métodos. Entre marzo y mayo de 2014, se realizaron encuestas estructuradas en hogares cafetaleros de dos paisajes vulnerables al cambio climático: Turrialba y Los Santos. El estudio se centró en las percepciones de los productores sobre los cambios en temperatura y lluvia, los impactos reportados como consecuencia de dichos cambios y las acciones de adaptación implementadas en las fincas. Resultados. Los resultados indicaron que el $98 \%$ de los productores percibió cambios en el clima, gran parte de ellos los asociaron con impactos sobre su producción de café, y el $60 \%$ de los encuestados realizó cambios en el manejo de sus cafetales para reducir los efectos del cambio climático. Las prácticas de adaptación más frecuentes fueron sembrar árboles e incrementar el uso de agroquímicos, en mayor medida en respuesta al incremento de temperatura. Conclusión. Este estudio destaca la necesidad de incrementar el apoyo técnico, financiero y político orientado a fomentar y diversificar la implementación de prácticas de adaptación entre pequeños caficultores e incrementar su resiliencia ante el cambio climático.

Palabras clave: adaptación al cambio climático, Adaptación basada en Ecosistemas, pérdidas de producción, políticas ambientales. 


\begin{abstract}
Introduction. Climate change will affect the distribution, productivity and profitability of coffee production in Central America, negatively impacting national economies and small farmer livelihoods. There is a need to understand how climate change affects small coffee farmers in the region in order to promote measures that allow them to cope with and adapt to these changes. Objective. The objective of this study was to describe Costa Rican small-scale coffee systems in two vulnerable agricultural landscapesand explorethe adaptation efforts that coffee farmers have implemented in these two coffee systems. Materials and methods. Structured surveys were conducted with coffeeproducing households in two highly vulnerable landscapes, Turrialba and Los Santos, in Costa Rica, from March-May 2014. The study was based on farmers' perceptions of changes in temperature and rain, reported impacts of these changes and the adaptation actions implemented at farm level. Results. Ninety-eight percentof farmers perceived changes in local climate and most of them related these changes with impacts on production (increase in pests and diseases, floweringproblems and other reported impacts). Sixty percentof the surveyed farmers had modified the management of their farms in order to reduce climate change impacts. The most common adaptation measures used by farmers were the planting of trees and the increased use of agrochemical inputs, mostly in response to perceptions of increasing temperatures. Conclusion. This study highlights the need for greater technical, financial and policy support to help smallholder coffee farmers implement adaptation practices and become more resilient to climate change.
\end{abstract}

Keywords: climate change adaptation, Ecosystem-based Adaptation, crop losses, environmental policies.

\title{
Introducción
}

La caficultura tiene gran relevancia económica y social en Costa Rica (Paiva, 2000). El café es el cultivo permanente con más extensión en el país (SEPSA, 2015); en 2014 representó el 5 \% del PIB agrícola y el 0,3 \% del PIB nacional (ICAFE, 2016), y constituyó el $11 \%$ de las exportaciones agrícolas (Alpízar et al., 2015). Este cultivo es altamente susceptible a los cambios en los patrones de temperatura y precipitación (Lin et al., 2008; Bunn et al., 2015) y al impacto de los eventos climáticos extremos, como las lluvias torrenciales o los huracanes (Schroth et al., 2009).

Los modelos climáticos indican que la región centroamericana experimentará incrementos de temperatura, cambios en los patrones de precipitación (Imbach et al., 2017) y aumento en la frecuencia y severidad de los eventos climáticos extremos en los próximos años (IPCC, 2012). Se espera que los cambios pronosticados reduzcan el área apta para el cultivo de café (Bunn et al., 2015; Ovalle-Rivera et al., 2015; Hannah et al., 2017), la productividad, la rentabilidad y la calidad del grano en América Central (Läderach et al., 2013). Estos cambios tendrán consecuencias económicas y sociales en los países productores, especialmente sobre las familias que dependen directa o indirectamente de este cultivo (CEPAL y CAC/SICA, 2014).

El cambio climático se suma a desafíos de tipo ambiental, productivos y de mercado que afronta la caficultura en la actualidad. Algunos de estos cambios (incremento de la temperatura, incertidumbre en el comienzo de las lluvias, entre otros), ya se están experimentando en la región (Aguilar et al., 2005; Magrin et al., 2014). Por la importancia del café en Costa Rica y los pronósticos de cambio e impactos sobre el cultivo, es relevante conocer si los caficultores han empezado a percibir cambios en temperatura y patrones de lluvia, si relacionan esos cambios con la productividad del café y si toman medidas específicas de manejo para adecuarse a las variaciones percibidas.

La percepción de cambios en el clima por productores, permite entender mejor el proceso de adaptación que está en marcha y sus debilidades, sin embargo, existe poca información sobre como los productores están percibiendo y experimentando el cambio climaticola región (Zuluaga et al., 2015). Parte de los estudios realizados 
en la región se centran en percepción, impactos y estrategias adaptativas en respuesta a eventos hidrometeorológicos extremos como huracanes, lluvias torrenciales o sequías (Tucker et al., 2010; Cruz-Bello et al., 2011; Ruiz-Meza, 2015). Otros estudios trabajan con múltiples factores de estrés, entre los que se incluyen la crisis del café, acceso a servicios básicos y el clima, entre otros (Schroth et al. 2009, Eakin et al., 2011, Frank et al. 2011, Eakin et al., 2014; Bacon et al., 2017). A nivel global, la literatura sobre la toma de decisiones de adaptación de pequeños productores aborda las percepciones en relación a eventos hidrometeorológicos extremos, variabilidad climática, tendencias del clima o varios de estos factores (Burnham y Ma, 2015).

Existen distintas estrategias no excluyentes para la adaptación de los sistemas agrícolas: medidas estructurales o físicas, como las obras de ingeniería, innovaciones tecnológicas, soluciones basadas en la naturaleza, opciones de tipo social, que incluyen educación, provisión de información para la toma de decisiones o acciones que promuevan cambios de comportamiento, y también opciones institucionales, entre ellas leyes y regulaciones, programas gubernamentales y mecanismos financieros (Noble et al., 2014). En este estudio, se hace énfasis en las medidas de adaptación a nivel de finca y, específicamente en el uso de prácticas de Adaptación basadas en Ecosistemas (AbE).

La Adaptación basada en Ecosistemas se fundamenta en el uso de la biodiversidad y los servicios ecosistémicos para incrementar la resiliencia de los sistemas frente al cambio climático (CBD, 2009). Se considera una opción de adaptación apropiada para pequeños productores por su viabilidad técnica y económica, y por los beneficios adicionales que aporta, tales como la reducción de emisiones (mitigación), el aumento de la seguridad alimentaria y la diversificación del ingreso familiar, el incremento de la conectividad y la biodiversidad a nivel de paisaje, entre otros co-beneficios, que mejoran los medios de vida de los pequeños productores y la sostenibilidad ambiental (Vignola et al., 2015).

El uso de sombra, de cercas vivas, de cobertura viva, la siembra en surcos a contorno y las chapias manuales son las prácticas AbE utilizadas en la caficultura incluidas en este estudio. Algunas de estas prácticas son frecuentes en las fincas (Harvey et al. 2017), ya que se han implementado en las últimas décadas como parte de las tendencias agroecológicas, agroforestales, etc. Sin embargo, no existe información sobre el uso de estas prácticas en respuesta a los cambios percibidos en el clima, ni de otras estrategias que son implementadas por los productores.

El objetivo de este trabajo fue describir los sistemas cafetaleros costarricenses de pequeña escala en dos zonas productivas, y presentar los esfuerzos de adaptación al cambio climático que realizan los productores.

\section{Materiales y métodos}

\section{Paisajes de estudio}

Se seleccionaron dos paisajes de estudio en Costa Rica con las siguientes características: (i) predominio de agricultura de pequeña escala, (ii) presencia de café como cultivo principal, (iii) elevada vulnerabilidad al cambio climático y (iv) baja capacidad adaptativa. Los paisajes seleccionados fueron Turrialba y Los Santos.

El paisaje cafetalero de Turrialba está ubicado en el cantón del mismo nombre, en la región productiva centraloriental de Costa Rica. El cantón tiene una extensión aproximada de $1642 \mathrm{~km}^{2}$, un rango altitudinal de 60 a 3800 msnm y una geomorfología montañosa (aproximadamente el $58 \%$ del territorio presenta pendientes mayores a los $30^{\circ}$ ). Los cafetales se distribuyen en cotas intermedias, entre 500 y $1300 \mathrm{msnm}$. Turrialba presenta un clima tropical húmedo con influencia atlántica, con una precipitación anual promedio de $2619 \mathrm{~mm}$ y una temperatura media anual de $21,7^{\circ} \mathrm{C}$. En 2011 la población era de 69616 personas, $43 \%$ de ellas distribuidas en la zona rural (MIDEPLAN y SICA, 2016). Las zonas de vida de Holdridge que ocupan la mayor parte del territorio son bosque pluvial y bosque muy húmedo (Holdridge, 1947).

El paisaje de Los Santos está conformado por los cantones de León Cortés, Tarrazú y Dota, y pertenece a la región cafetalera Tarrazú, en la región productiva central-occidental. El rango altitudinal va de 100 a 3400 msnm. El 
café se distribuye en la zona media y media-alta, entre 1000 y 2300 msnm, aproximadamente. En 2011, la población era de 35428 personas, $43 \%$ de ellas distribuidas en la zona rural (INDER, 2016). El clima es biestacional con influencia climática del Pacífico, con estaciones secas y lluviosas bien definidas y una temperatura promedio entre 17,5 y $20^{\circ} \mathrm{C}$ en los distintos cantones. La zona es reconocida internacionalmente por su producción de café de altura con gran calidad de taza (ICAFE, s.f.). Las zonas de vida de Holdridge que ocupan la mayor parte del territorio son bosque muy húmedo y bosque pluvial (Holdridge, 1947).

\section{Muestreo y recolección de datos}

La información que se presenta en este estudio fue recolectada entre marzo y mayo de 2014. Se seleccionaron aleatoriamente hogares caficultores y se entrevistó a 144 jefes de hogar con capacidad de tomar decisiones sobre el manejo de la finca en Turrialba y 151 en Los Santos. Se usó como marco muestral el último censo cafetalero disponible. En el caso de Turrialba, el último censo cafetalero databa de 2003 y señalaba un total de 1042 productores individuales (no compañías). En Los Santos, el último censo cafetalero disponible data de 2006 y el total de productores individuales era de 1706 (INEC, 2007).

La implementación de la encuesta se realizó en persona por un equipo de encuestadores entrenados y un supervisor de campo. Antes de su implementación, la encuesta fue pilotada en campo y aprobada por el Comité de Revisión Ética Interno de Conservación Internacional. De esta forma se verificó que la encuesta siguiera la normativa institucional para la participación libre e informada de los productores (Buppert y McKeehan, 2013). Los datos se recolectaron con dispositivos electrónicos (tabletas) por medio del software Survey-CTO.

En este estudio, se presentan los resultados de la encuesta realizada a 295 pequeños caficultores costarricenses. Debido a la falta de acuerdo sobre la definición de pequeño productor (Nagayets, 2005; Conway, 2011), se solicitó a los encuestados que se autodefiniesen (o no) como pequeños productores. De esta forma, los resultados corresponden a pequeños productores según el estándar de cada paisaje, un total de 144 productores en Turrialba (122 hombres, 22 mujeres) y 151 en Los Santos (136 hombres, 15 mujeres).

\section{Información recolectada y análisis realizados}

La información que se describe en este estudio fue recolectada con una encuesta estructurada, que contenía 400 preguntas de selección única o selección múltiple. La encuesta, así como la metodología utilizada para la recolección de datos se detalla en Alpizar et al. (2019). Para describir los sistemas cafetaleros de los paisajes, se realizaron preguntas sobre las características socioeconómicas de los jefes de hogar y de los hogares. Además, se recolectó información sobre un conjunto amplio de aspectos del sistema productivo, como la presencia de ganado, granos básicos y huertos caseros. Para caracterizar el manejo del café, se hicieron preguntas sobre el uso de un conjunto amplio de prácticas: chapias, uso de agroquímicos, poda, deshije y otros. Finalmente, se preguntó sobre las variedades de café utilizadas. Estas preguntas se hicieron a cada entrevistado, no así las preguntas de otras secciones, que solamente se realizaron a aquellos encuestados que dieron una respuesta específica a una pregunta previa o pregunta de seguimiento (Cuadro 1).

Como fue mencionado en la introducción, el uso de prácticas de Adaptación basadas en Ecosistemas es de particular interés por tratarse de prácticas útiles para lidiar con el cambio climático. En la encuesta se recolectó información de las siguientes prácticas basadas en ecosistemas: surcos a contorno, cercas vivas, sombra, poda de la sombra y chapias manuales. Para cada una de estas prácticas, se hizo una serie de preguntas de seguimiento a los encuestados que reportaron su uso. Los resultados se analizaron indicando el número de observaciones por paisaje y práctica (Cuadro 1). Las preguntas de seguimiento buscaban describir cuándo se implementó la práctica por 
Cuadro 1. Información recolectada y variación del tamaño de la muestra según la condicionalidad de las preguntas de la encuesta realizada a pequeños caficultores en Turrialba y Los Santos, Costa Rica. 2014.

Table 1. A summary of the information collected in the surveys of smallholder coffee farmers in Turrialba and Los Santos, and variation in sample size for each section, based on the conditionality of survey questions. Costa Rica. 2014.

\begin{tabular}{|c|c|c|c|c|}
\hline \multirow{2}{*}{ Quién responde la pregunta } & \multirow{2}{*}{ Qué información se presenta } & \multicolumn{2}{|c|}{ Observaciones } & \multirow{2}{*}{ Ubicación } \\
\hline & & Turrialba & Los Santos & \\
\hline Todos los productores & $\begin{array}{l}\text { Caracterización de jefes, hogares, fincas y } \\
\text { manejo del café }\end{array}$ & 144 & 151 & Cuadro 2 \\
\hline Todos los productores & $\begin{array}{l}\text { Variedades de café utilizadas y razones de } \\
\text { cambio de variedades }\end{array}$ & 144 & 151 & Cuadro 3 \\
\hline $\begin{array}{l}\text { Los productores que usan cada práctica } \\
\text { contestan las preguntas de seguimiento }\end{array}$ & $\begin{array}{l}\text { Uso de prácticas AbE } \\
\text { - Surcos a contorno............. } \\
\text { - Cercas vivas } \ldots \ldots \ldots \ldots \ldots \ldots \ldots \ldots \\
\text { - Sombra........................ } \\
\text { - Poda de la sombra............. } \\
\text { - Chapias manuales............ }\end{array}$ & $\begin{array}{l}111 \\
127 \\
139 \\
134 \\
127\end{array}$ & $\begin{array}{l}139 \\
118 \\
149 \\
148 \\
148\end{array}$ & Cuadro 4 \\
\hline $\begin{array}{l}\text { Todos los productores. La diferencia con } \\
\text { respecto a la muestra total se debe a no } \\
\text { respuesta de algunos productores. }\end{array}$ & Problemas de producción en el cafetal & 143 & 147 & Figura 2 \\
\hline $\begin{array}{l}\text { Sólo los productores que perciben cambios } \\
\text { en temperatura/lluvia, contestan la pregunta } \\
\text { de seguimiento: ¿cuáles cambios ha } \\
\text { percibido en T/Ll? }\end{array}$ & $\begin{array}{l}\text { Percepciones de cambios en: } \\
\text { - Temperatura................... } \\
\text { - Lluvia.......................... }\end{array}$ & $\begin{array}{l}138 \\
135\end{array}$ & $\begin{array}{l}145 \\
141\end{array}$ & Cuadro 5 \\
\hline $\begin{array}{l}\text { Sólo los productores que reportan que } \\
\text { cambios en el clima han afectado la } \\
\text { producción de café. }\end{array}$ & $\begin{array}{l}\text { Cambios en el clima que han afectado la } \\
\text { producción de café }\end{array}$ & 111 & 98 & Cuadro 6 \\
\hline $\begin{array}{l}\text { Solo los productores que reportan que } \\
\text { han implementado cambios de manejo en } \\
\text { respuesta a los cambios percibidos en el clima }\end{array}$ & $\begin{array}{l}\text { Prácticas de manejo de finca que ha } \\
\text { implementado debido a cambios percibidos } \\
\text { en temperatura y lluvia }\end{array}$ & 63 & 115 & Cuadro 7 \\
\hline
\end{tabular}

primera vez y si se modificó en los últimos diez años. Adicionalmente, se preguntó sobre los beneficios percibidos de su uso.

La caracterización de los sistemas productivos concluyó con una pregunta sobre los problemas experimentados en la producción de café. Esta era una pregunta de respuesta múltiple que fue hecha a todos los productores. Sin embargo, algunos encuestados dijeron no tener ningún problema en el cafetal, por lo que la respuesta se reportó para 143 productores en Turrialba y 147 productores en Los Santos (Cuadro 1).

En este estudio también se describen las percepciones de los encuestados sobre cambios en el clima que les han afectado, así como las acciones que implementaron en respuesta a esas percepciones. Con respecto a cambios en el clima, se preguntó específicamente a cada encuestado si percibió cambios en temperatura en los diez años anteriores a la encuesta. Si la respuesta era positiva, se preguntaba: (a) cuáles fueron los cambios percibidos, y (b) si había implementado algún cambio en el manejo de la finca debido a las percepciones de cambio en temperatura. Si la respuesta a la segunda pregunta era positiva, se preguntaba cuáles alternativas de manejo de finca fueron implementados (Cuadro 1). Un conjunto similar de preguntas se hizo para variaciones percibidas en patrones de lluvia.

Para profundizar en la relación entre cambios en temperatura y lluvia con la producción de café, se planteó una pregunta general para saber si el entrevistado consideraba que alguno de los cambios en el clima había afectado 
negativamente su producción de café. En caso de que la respuesta fuera positiva, se preguntaba cuáles cambios en el clima habían afectado la producción. Se registró cualquier respuesta obtenida, que podía incluir alteraciones en temperatura, lluvia o la mención de eventos hidrometeorológicos extremos. El Cuadro 1 refleja la cantidad de respuestas positivas y observaciones en la pregunta de seguimiento en cada paisaje.

La información recolectada se describe por medio de tablas e indicadores estadísticos. Los resultados obtenidos se discuten con base en investigaciones realizadas a nivel regional (Cruz-Bello et al., 2011; Eakin et al., 2014; RuizMeza, 2015; Zuluaga et al., 2015; Bacon et al., 2017; Harvey et al., 2017), y se plantean ideas clave para promover la adaptación del sector cafetalero costarricense al cambio climático.

\section{Resultados}

\section{Caracterización de productores, fincas y manejo del café}

Se encontraron diferencias a nivel de finca, hogar y recursos disponibles entre los hogares productores de café (C. arabica) en Turrialba y Los Santos (Cuadro 2).

Cuadro 2. Características de las fincas, de los jefes de hogar y los hogares de los caficultores encuestados en los paisajes de Turrialba y Los Santos, Costa Rica. 2014.

Table 2. Characteristics of the farms, household heads, and households of coffee farmers surveyed in Turrialba and Los Santos, Costa Rica. 2014.

\begin{tabular}{|c|c|c|c|c|}
\hline \multirow{3}{*}{$\begin{array}{l}\text { Número de observaciones } \\
\text { Características de la finca }\end{array}$} & \multicolumn{2}{|c|}{ Turrialba } & \multicolumn{2}{|c|}{ Los Santos } \\
\hline & \multicolumn{2}{|c|}{144} & \multicolumn{2}{|c|}{151} \\
\hline & Promedio & DE & Promedio & DE \\
\hline Tamaño finca (ha) & 2,63 & $\pm 2,43$ & 5,45 & $\pm 7,89$ \\
\hline Número de parcelas (cantidad) & 1,31 & $\pm 0,59$ & 1,83 & $\pm 1,21$ \\
\hline Distancia al hogar $(\mathrm{km})$ & 0,77 & $\pm 1,39$ & 2,25 & $\pm 3,04$ \\
\hline Altitud (msnm) & 862,19 & $\pm 152,21$ & 1601,4 & $\pm 143,88$ \\
\hline \multicolumn{5}{|l|}{ Características del jefe de hogar } \\
\hline Jefatura femenina $(\%)$ & 15,28 & & 9,93 & \\
\hline Edad (años) & 58,15 & $\pm 13,94$ & 51,88 & $\pm 12,79$ \\
\hline Experiencia (años) & 39,58 & $\pm 18,14$ & 34,71 & $\pm 14,66$ \\
\hline Más de seis años de educación oficial (\%) & 15,28 & & 23,17 & \\
\hline \multicolumn{5}{|l|}{ Características del hogar } \\
\hline Tamaño del hogar (miembros) & 3,36 & $\pm 1,57$ & 3,68 & $\pm 1,55$ \\
\hline Hogares con migrantes permanentes $(\%)$ & 35,42 & & 21,19 & \\
\hline Trabajo en finca (n personas) & 1,43 & $\pm 0,82$ & 1,60 & $\pm 0,92$ \\
\hline Trabajo fuera de finca (n personas) & 1,03 & $\pm 0,92$ & 0,81 & $\pm 0,86$ \\
\hline Tiene ganadería $(\%)$ & 26,39 & & 28,47 & \\
\hline Tiene aves de patio (\%) & 29,86 & & 45,70 & \\
\hline Tiene huerto casero (\%) & 22,22 & & 50,33 & \\
\hline Cultiva granos básicos $(\%)$ & 9,03 & & 3,31 & \\
\hline Vehículo (carro o motocicleta) (\%) & 49,31 & & 90,07 & \\
\hline Pertenencia a organización (\%) & 29,86 & & 39,07 & \\
\hline
\end{tabular}

DE: desviación estándar / DE: standard deviations. 
Los paisajes difirieron en el manejo de café. En general, el manejo era menos intensivo en Turrialba que en Los Santos. En Turrialba se realizaban en promedio 2,3 chapias, 1,6 aplicaciones de fertilizante, 1,5 de fungicida, 1,6 de herbicida y se deshijaban las plantas de café 1,2 veces por año. El $53 \%$ de los productores sembraba su café junto con otros cultivos. Casi todos los productores (98\%) entregaban su café a un beneficio privado; muy pocos trabajaban con un comprador independiente ( $1 \%$ o con una cooperativa de productores $(1 \%)$. En promedio, los productores manejaban 1,5 variedades de café por finca. La variedad más extendida en los cafetales de Turrialba era Caturra, seguido por Catuai, Catimor y Costa Rica 95 (Cuadro 3). Otras variedades estaban escasamente representadas $(<10 \%)$. En el último decenio, las variedades más introducidas eran Caturra, Catuai, Catimor y Costa Rica 95 con el fin de incrementar la resistencia a plagas y patógenos y a incrementar la productividad.

Cuadro 3. Diversidad de variedades de café utilizadas en las fincas de café de Turrialba y Los Santos, y razones de cambio de variedades. 2004 - 2014. Costa Rica.

Table 3. Coffee varieties cultivated in Turrialba and Los Santos farms, and reasons for changing varieties. 2004 - 2014. Costa Rica.

\begin{tabular}{|c|c|c|}
\hline & Turrialba & Los Santos \\
\hline Productores de café (n) & 144 & 151 \\
\hline Promedio de variedades por finca & 1,55 & 2,17 \\
\hline Tiene $>1$ variedad & $43 \%$ & $78 \%$ \\
\hline Tiene $>2$ variedades & $9 \%$ & $30 \%$ \\
\hline Cambió variedades & $22 \%$ & $18 \%$ \\
\hline \multicolumn{3}{|l|}{ Variedades sembradas } \\
\hline Caturra & $85 \%$ & $84 \%$ \\
\hline Catuai & $29 \%$ & $83 \%$ \\
\hline Catimor & $16 \%$ & $25 \%$ \\
\hline Costa Rica - 95 & $11 \%$ & $6 \%$ \\
\hline Geisha & $0 \%$ & $5 \%$ \\
\hline Híbrido tico (no definidos) & $6 \%$ & $6 \%$ \\
\hline Otros & $3 \%$ & $8 \%$ \\
\hline \multicolumn{3}{|l|}{ Razones de cambio de variedades de café } \\
\hline Productores que cambiaron de variedad & 31 & 27 \\
\hline Resistencia a plagas o patógenos & $52 \%$ & $37 \%$ \\
\hline Aumentar productividad & $42 \%$ & $30 \%$ \\
\hline Razones de mercado (e.g., precios) & $10 \%$ & $44 \%$ \\
\hline Resistencia a frío & $0 \%$ & $7 \%$ \\
\hline Resistencia a sequía & $0 \%$ & $4 \%$ \\
\hline Recibe incentivo para cambiar & $0 \%$ & $0 \%$ \\
\hline Otra & $26 \%$ & $11 \%$ \\
\hline
\end{tabular}

En Los Santos los productores realizaban un promedio de 2,7 chapias al año, 2,7 aplicaciones de fertilizante, 3,3 de fungicida, 1,2 de herbicida y se deshijaba 1,1 veces por año. El $43 \%$ sembraban el café acompañado de otros cultivos. El $17 \%$ de las fincas estaban certificadas ${ }^{6}$ y el $7 \%$ producían café orgánico. La mayor parte de los productores $(79 \%)$ entregaban su café a una cooperativa y un $29 \%$ trabajaban con un beneficio privado. Tanto la variedad Caturra como Catuai eran muy utilizadas en este paisaje; en menor medida se encontraban Catimores

\footnotetext{
6 No se especificó qué certificación tenían, ni quién les dio el certificado orgánico.
} 
y nuevas variedades como Geisha e híbridos costarricenses (no definidos, probablemente F1) $(<10 \%)$ (Cuadro 3). En el último decenio, las variedades más introducidas eran Caturra, Catuai y Catimor, para el incremento de la resistencia del café a plagas y patógenos, su productividad y un mejor acceso al mercado. La diversidad de variedades en las fincas de este paisaje fue mayor que en Turrialba, ya que había un promedio de 2,2 variedades por finca y el $78 \%$ de los productores tenían más de una variedad sembrada.

En ambos paisajes de estudio se manejaba el café con sombra, cuya composición y estructura varió entre paisajes. En Turrialba, el $34 \%$ de las fincas manejaban una especie de sombra, el $48 \%$ dos y el $15 \%$ tres o más especies. Entre las especies más comunes se encontraban el poró (Erythrina poeppigiana), presente en el $83 \%$ de las fincas, musáceas como el banano (Musa acuminata), el plátano (M. paradisiaca) y el guineo (Musa sp.) en el $55 \%$ de las fincas, el laurel (Cordia alliodora) en el $21 \%$ de las fincas, y de forma minoritaria otras especies frutales y maderables.

En Los Santos, el $51 \%$ de los productores tenían dos especies de sombra y el $40 \%$ tres o más. Las especies más comunes eran los porós (Erythrina sp.), presente en el $90 \%$ de las fincas, y las musáceas como el banano, el plátano y el guineo, que crecían en las fincas de café del $93 \%$ de los encuestados. También abundaban otras especies de frutales, en particular el aguacate (Persea americana) que estaba presente en el $30 \%$ de las fincas.

\section{Uso de prácticas basadas en ecosistemas y problemas reportados en los cafetales}

La información recolectada sobre cuatro prácticas de Adaptación basadas en Ecosistemas (surcos a contorno, cercas vivas, uso de árboles de sombra y chapias manuales o limpias) mostró que estas prácticas eran utilizadas en forma tradicional por la mayoría de los productores en ambos paisajes de forma tradicional (las implementaron hace más de diez años) (Cuadro 4). El manejo de estas prácticas era dinámico, pues entre 2004 y 2014 los productores realizaron cambios en estas prácticas (Cuadro 4): sembraron más cercas vivas y más árboles de sombra e incrementaron la diversidad de especies. Además, algunos productores incrementaron la intensidad de poda y otros la redujeron; aplicaron menos herbicida en este periodo como consecuencia del uso de chapias manuales y limpias. Los productores percibieron los beneficios del uso de las prácticas, principalmente de tipo adaptativo, mejoramiento de las condiciones del suelo, incremento de la rentabilidad, entre otros (Figura 1). Los beneficios adaptativos de protección frente a lluvias intensas se asoció al uso de surcos a contorno y el incremento de la tolerancia a sequía y temperaturas extremas al uso de sombra. El mejoramiento de las condiciones del suelo se atribuyó al uso de cercas vivas y sombra. Las chapias tuvieron un papel importante en la provisión de servicios de regulación y las cercas vivas en la provisión de productos adicionales al café como leña, madera o frutos.

El $99 \%$ de los caficultores de Turrialba y el $97 \%$ de los caficultores de Los Santos experimentaron algún problema en la producción de café en los últimos diez años. Los problemas reportados pudieron no estar relacionados con cambios en el clima. En ambos paisajes el problema reportado con más frecuencia fue el daño provocado por patógenos, particularmente por la roya del cafeto (Hemileia vastatrix) y, en menor medida, por el ojo de gallo (Mycena citricolor). Otros daños importantes se relacionaron con la dispersión de la floración en un periodo más largo y con la muerte de plantas (Figura 2).

\section{Percepciones de cambio climático e impactos sobre el café}

El $98 \%$ de todos los productores encuestados dijo haber percibido un cambio en el clima a nivel local en los últimos diez años (entre 2004 y 2014). En Turrialba, el $96 \%$ observó cambios relacionados con la temperatura y el $94 \%$ con la precipitación; mientras que, en Los Santos el $96 \%$ y el $93 \%$, respectivamente, reportaron dichos cambios. La percepción del cambio era generalizada y consistente respecto a temperatura, no así con los patrones de cambio de precipitación (Cuadro 5). 


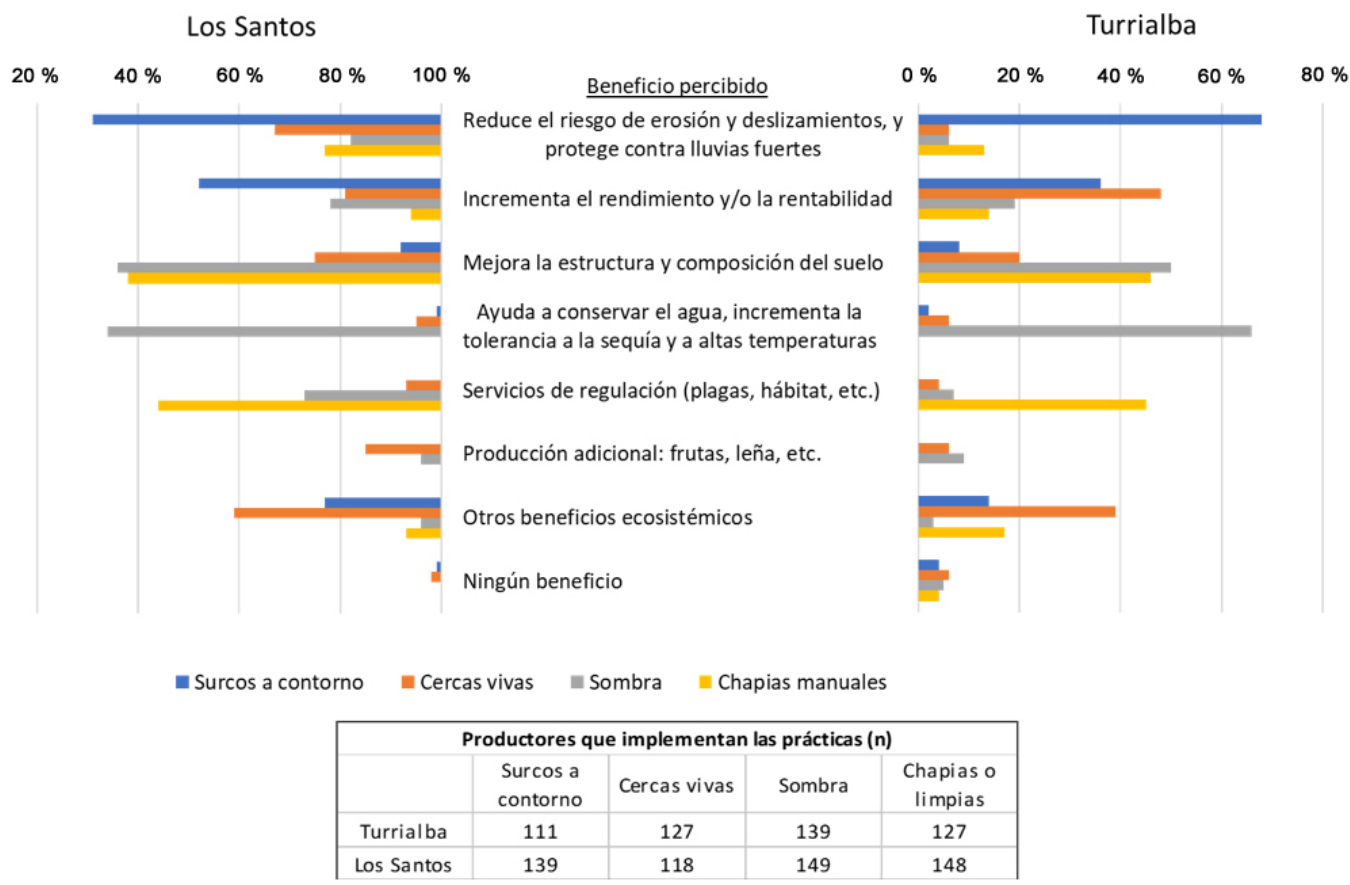

Figura 1. Beneficios del uso de las prácticas de Adaptación basada en Ecosistemas (AbE) percibidos por los productores de café en Turrialba y Los Santos. Costa Rica. 2014.

Figure 1. Benefits from using the Ecosystem-based Adaptation (EbA) practices perceived by coffee farmers in Turrialba and Los Santos. Costa Rica. 2014.

Cuadro 4. Productores de café, en Turrialba y Los Santos (Costa Rica), que utilizaban cada práctica de Adaptación basada en Ecosistemas (AbE), el porcentaje de ellos que comenzó a utilizarlas en los últimos diez años y los cambios que realizaron en el manejo de la práctica en este periodo. 2004-2014.

Table 4. Number of coffee farmers from Turrialba and Los Santos (Costa Rica) that used each of the Ecosystem-based Adaptation $(\mathrm{EbA})$ practices, the percentage of them that began to use them during the last ten years, and the changes made in the management of the practice during this period. 2004-2014.

\begin{tabular}{|c|c|c|c|c|c|c|c|c|}
\hline & \multicolumn{4}{|c|}{ Turrialba } & \multicolumn{4}{|c|}{ Los Santos } \\
\hline & $\begin{array}{l}\text { Surcos a } \\
\text { contorno }\end{array}$ & $\begin{array}{c}\text { Cercas } \\
\text { vivas }\end{array}$ & Sombra & $\begin{array}{l}\text { Chapias } \\
\text { manuales }\end{array}$ & $\begin{array}{l}\text { Surcos a } \\
\text { contorno }\end{array}$ & $\begin{array}{c}\text { Cercas } \\
\text { vivas }\end{array}$ & Sombra & $\begin{array}{c}\text { Chapias } \\
\text { manuales }\end{array}$ \\
\hline $\mathbf{N}$ productores & 111 & 127 & 139 & 127 & 139 & 118 & 149 & 148 \\
\hline Implementa entre 2004-2014 (\%) & 7 & 21 & 19 & 22 & 4 & 28 & 13 & 25 \\
\hline \multicolumn{9}{|c|}{ Cambios de manejo en 10 años } \\
\hline Incrementa el uso & - & $36 \%$ & $35 \%$ & $11 \%$ & - & $42 \%$ & $63 \%$ & $26 \%$ \\
\hline Reduce el uso & - & $5 \%$ & $14 \%$ & $12 \%$ & - & $2 \%$ & $2 \%$ & $6 \%$ \\
\hline Diversifica la práctica & - & $24 \%$ & $14 \%$ & - & - & $22 \%$ & $17 \%$ & - \\
\hline Reduce la diversidad en práctica & - & $2 \%$ & $2 \%$ & - & - & $0 \%$ & $1 \%$ & - \\
\hline Poda con más frecuencia o intensidad & - & $17 \%$ & $21 \%$ & - & - & $9 \%$ & $7 \%$ & - \\
\hline $\begin{array}{l}\text { Poda con menos frecuencia o } \\
\text { intensidad }\end{array}$ & - & $5 \%$ & $12 \%$ & - & - & $2 \%$ & $6 \%$ & - \\
\hline Disminuye aplicación de herbicidas & - & - & - & $48 \%$ & - & - & - & $62 \%$ \\
\hline Otras & - & $13 \%$ & $32 \%$ & - & - & $8 \%$ & $28 \%$ & - \\
\hline
\end{tabular}




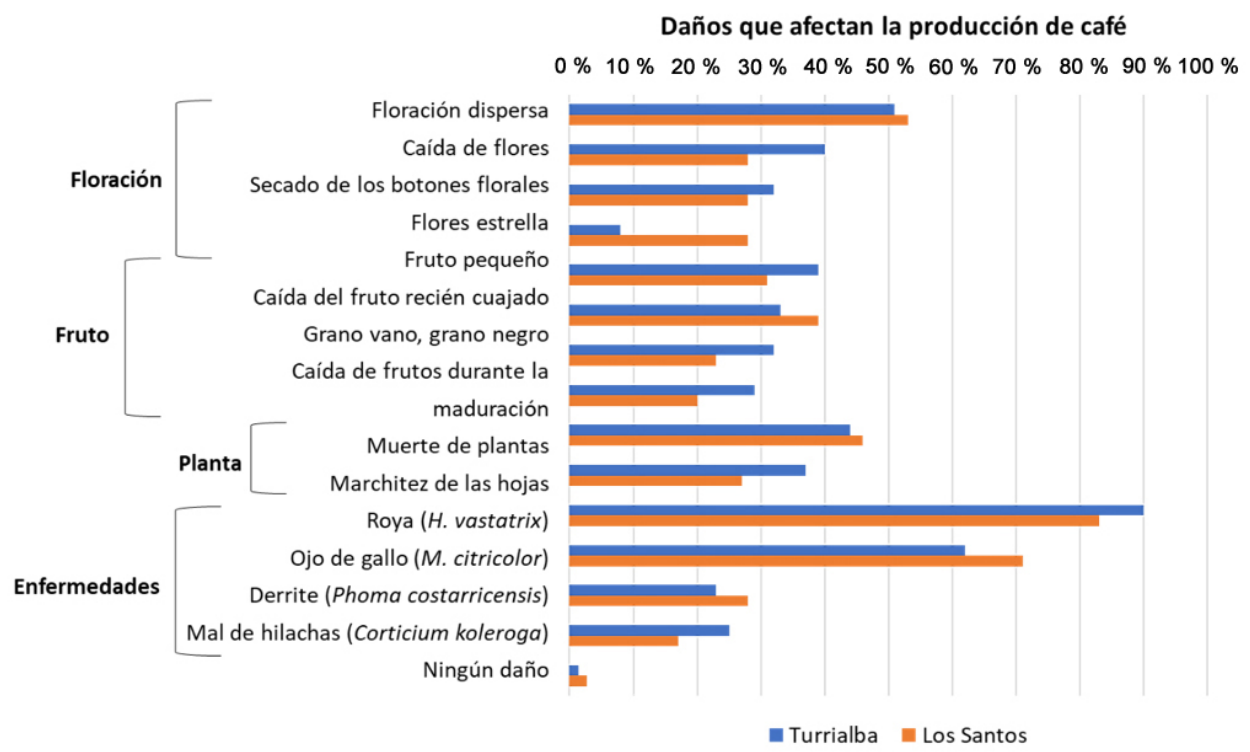

Figura 2. Problemas de producción reportados por los productores de café de Turrialba (n=144) y de Los Santos ( $\mathrm{n}=151)$, Costa Rica, durante los años 2004-2014, independientemente de si creían que el problema estaba asociado al cambio del clima o no.

Figure 2. Coffee production problems reported by farmers in Turrialba $(n=144)$ and in Los Santos ( $n=151)$, Costa Rica during the years 2004-2014, regardless of whether they believed that these problems were liked to climate change or not.

Cuadro 5. Cambios percibidos por los productores en el clima local durante los últimos diez años (2004-2014) en Turrialba y Los Santos, Costa Rica.

Table 5. Changes perceived by coffee farmers in local climate during the last ten years (2004-2014) in Turrialba and Los Santos, Costa Rica.

\begin{tabular}{lcc}
\hline & Turrialba & Los Santos \\
\hline Productores que perciben cambio en temperatura & $\mathbf{1 3 8}$ & $\mathbf{1 4 5}$ \\
Ha incrementado & $98 \%$ & $97 \%$ \\
Ha disminuido & $0 \%$ & $4 \%$ \\
Mayor rango de variación de temperatura & $9 \%$ & $4 \%$ \\
Menor rango de variación de temperatura & $1 \%$ & $2 \%$ \\
\hline Productores que perciben cambio en precipitación & $\mathbf{1 3 5}$ & $\mathbf{1 4 1}$ \\
Llueve más & $4 \%$ & $67 \%$ \\
Llueve menos & $67 \%$ & $38 \%$ \\
Llueve más intenso & $20 \%$ & $44 \%$ \\
Cambio en los patrones de lluvia & $50 \%$ & $1 \%$ \\
\hline
\end{tabular}

El $78 \%$ de los productores de café de Turrialba y el $67 \%$ de Los Santos consideraron que los cambios del clima (principalmente el incremento de temperatura y en menor medida la reducción de la lluvia) han afectado negativamente la producción de café en los últimos años (Cuadro 6). Los cambios reportados son muy relevantes, ya que las percepciones personales son las que definieron las decisiones a nivel de familia y de finca, como fue el 
Cuadro 6. Cambios del clima que afectan negativamente la producción de café, según percepciones de los caficultores de Turrialba y Los Santos, Costa Rica. 2004-2014.

Table 6. Changes in climate that negatively affect coffee production, based on the perceptions of coffee farmers of Turrialba and Los Santos, Costa Rica. 2004-2014.

\begin{tabular}{lcc}
\hline & Turrialba & Los Santos \\
\hline $\begin{array}{l}\text { Productores que consideran que los cambios del clima han } \\
\text { afectado negativamente la producción de café }\end{array}$ & $\mathbf{1 1 1}$ & $\mathbf{9 8}$ \\
Incremento de temperatura & $61 \%$ & $76 \%$ \\
Menos lluvia anual & $27 \%$ & $41 \%$ \\
Incertidumbre en el comienzo de las lluvias & $25 \%$ & $27 \%$ \\
Lluvia concentrada en menos días (más intensa) & $12 \%$ & $13 \%$ \\
Sequías más prolongadas & $33 \%$ & $15 \%$ \\
\hline
\end{tabular}

caso de las decisiones adaptativas que se pretendieron identificar en este trabajo (aún considerando que se requieren series de datos más largas para definir cambios significativos en las variables climáticas). Además, la variación en los patrones climáticos, así como los efectos negativos de estos en la cosecha, fueron identificables por los productores con base en su experiencia y en los indicadores de cambio de cada cultivo (por ejemplo, variables que favorecen la formación de yemas en determinado momento del año).

\section{Cambios de manejo en respuesta a las variaciones percibidas en temperatura y lluvia}

El porcentaje de productores que implementó medidas para adaptar sus sistemas productivos al cambio percibido en el clima fue relativamente bajo, a pesar de que la percepción de cambio era generalizada y los productores la asociaron a impactos en sus cosechas. Entre los productores de Turrialba que reportaron cambios en el clima, solamente el $45 \%$ había realizado acciones para adaptar sus cultivos a las nuevas condiciones climáticas, mientras que, en Los Santos el $78 \%$ de los productores reportaron implementar medidas de adaptación al cambio climático.

Los cambios de manejo más frecuentes en ambos paisajes en respuesta a las alteraciones percibidas, fueron la incorporación de árboles en la finca (50\%), el incremento del uso de pesticidas, herbicidas, fungicidas (24 \%) y fertilizantes $(15 \%)$, y la introducción de prácticas de manejo y conservación de suelos (15\%) (Cuadro 7). Estas prácticas se utilizaban mayoritariamente en respuesta a los cambios de temperatura, a excepción del manejo y conservación de agua, que respondía a los cambios en precipitación. La implementación de prácticas tecnológicas (tales como introducción de variedades mejoradas, riego, etc.) era baja y diferente entre paisajes: en Turrialba optaban por aplicar riego y en Los Santos por adecuar el calendario agrícola. Algunos productores (13\%) optaron por reducir el uso de pesticidas, herbicidas y fungicidas. Las prácticas relacionadas con diversificación (cultivos, animales) eran escasamente implementadas en ambos paisajes (Cuadro 7). En promedio, los productores de ambos paisajes implementaban entre 1 y 2 prácticas de adaptación. 
Cuadro 7. Cambios de manejo del cafetal en respuesta a los cambios percibidos en temperatura y precipitación en Turrialba y Los Santos, Costa Rica. 2004-2014.

Table 7. Changes in coffee management practices in response to perceived changes in climate in Turrialba and Los Santos, Costa Rica. 2004-2014.

\begin{tabular}{|c|c|c|c|c|c|c|c|c|c|c|}
\hline \multirow{4}{*}{$\begin{array}{l}\text { Cambios de manejo } \\
\text { Productores que hacen cambios en el manejo en } \\
\text { respuesta a cambios percibidos en el clima }\end{array}$} & \multicolumn{4}{|c|}{ Turrialba } & \multicolumn{4}{|c|}{ Santos } & \multirow{2}{*}{\multicolumn{2}{|c|}{ Total }} \\
\hline & \multicolumn{2}{|c|}{ Temp. } & \multicolumn{2}{|c|}{ Precip. } & \multicolumn{2}{|c|}{ Temp. } & \multicolumn{2}{|c|}{ Precip. } & & \\
\hline & \multicolumn{2}{|c|}{46} & \multicolumn{2}{|c|}{44} & \multicolumn{2}{|c|}{92} & \multicolumn{2}{|c|}{86} & \multicolumn{2}{|c|}{178} \\
\hline & $\mathbf{n}$ & $(\%)$ & $\mathbf{n}$ & $(\%)$ & $\mathbf{n}$ & $(\%)$ & $\mathbf{n}$ & $(\%)$ & $\mathbf{n}$ & $(\%)$ \\
\hline Plantar árboles dentro de la finca & 12 & 26 & 7 & 16 & 60 & 65 & 35 & 41 & 89 & 50 \\
\hline $\begin{array}{l}\text { Cambiar las prácticas de manejo del cultivo (respuesta } \\
\text { general, si no menciona detalles) }\end{array}$ & 10 & 22 & 9 & 20 & 17 & 18 & 16 & 19 & 45 & 25 \\
\hline Aplicar más pesticidas, herbicidas y fungicidas & 6 & 13 & 5 & 11 & 19 & 21 & 17 & 20 & 42 & 24 \\
\hline Aplicar más fertilizantes & 8 & 17 & 5 & 11 & 13 & 14 & 9 & 10 & 27 & 15 \\
\hline $\begin{array}{l}\text { Introducir prácticas de manejo y conservación de } \\
\text { suelo }\end{array}$ & 2 & 4 & 5 & 11 & 6 & 7 & 17 & 20 & 26 & 15 \\
\hline Aplicar menos pesticidas, herbicidas y fungicidas & 5 & 11 & 5 & 11 & 8 & 9 & 8 & 9 & 23 & 13 \\
\hline Otros & 8 & 17 & 2 & 5 & 7 & 8 & 1 & 1 & 17 & 10 \\
\hline $\begin{array}{l}\text { Introducir prácticas de manejo y conservación de } \\
\text { agua }\end{array}$ & 0 & 0 & 4 & 9 & 3 & 3 & 5 & 6 & 10 & 6 \\
\hline Cambiar el calendario agrícola & 3 & 0 & 0 & 2 & 3 & 3 & 3 & 9 & 10 & 6 \\
\hline Aplicar menos fertilizantes & 2 & 7 & 5 & 0 & 1 & 3 & 1 & 3 & 8 & 5 \\
\hline Regar los cultivo & 0 & 4 & 1 & 11 & 3 & 1 & 8 & 1 & 7 & 4 \\
\hline Sembrar cultivos que antes no sembraba & 3 & 7 & 1 & 2 & 1 & 1 & 0 & 0 & 4 & 2 \\
\hline Diversificar la producción en la finca & 2 & 4 & 0 & 0 & 1 & 1 & 0 & 0 & 3 & 2 \\
\hline Dejar de cultivar ciertas zonas de la finca & 2 & 4 & 2 & 5 & 0 & 0 & 0 & 0 & 2 & 1 \\
\hline Cortar árboles dentro de la finca & 1 & 2 & 1 & 2 & 1 & 1 & 0 & 0 & 2 & 1 \\
\hline Cambiar la variedad de cultivos sembrados & 0 & 0 & 1 & 2 & 1 & 1 & 0 & 0 & 2 & 1 \\
\hline Restaurar áreas degradadas en la finca & 0 & 0 & 1 & 2 & 1 & 1 & 0 & 0 & 2 & 1 \\
\hline Introducir animales que antes no tenía & 0 & 0 & 0 & 0 & 0 & 0 & 0 & 0 & 0 & 0 \\
\hline Aumentar la cantidad de animales & 0 & 0 & 0 & 0 & 0 & 0 & 0 & 0 & 0 & 0 \\
\hline Reducir la cantidad de animales & 0 & 0 & 0 & 0 & 0 & 0 & 0 & 0 & 0 & 0 \\
\hline Promedio de prácticas implementadas $(\mathrm{DE})^{*}$ & \multicolumn{2}{|c|}{$1,3(0,5)$} & \multicolumn{2}{|c|}{$1,2(0,5)$} & \multicolumn{2}{|c|}{$1,4(0,7)$} & \multicolumn{2}{|c|}{$1,3(0,7)$} & \multicolumn{2}{|c|}{$1,6(0,9)$} \\
\hline
\end{tabular}

* Sin contar respuesta general / Excluding general answer. DE: desviación estándar / DE: standard deviations.

\section{Discusión}

Este estudio muestra que los pequeños productores de café en Costa Rica son vulnerables al cambio climático, experimentan los impactos en sus sistemas productivos y en sus medios de vida, y han comenzado a implementar acciones para enfrentar dichos impactos, sin embargo, es necesario promover la inclusión de acciones de adaptación para incrementar su uso. 
En ambos paisajes de estudio los pequeños caficultores percibieron cambios en el clima. La mayor parte de estos habían experimenta cambios en temperatura y lluvia, lo cual dificulta la planificación de sus actividades productivas. Las percepciones de los productores reflejaron los cambios que se están observando en el clima a nivel regional (Aguilar et al., 2005; Donat et al., 2013), incluyendo variaciones en los patrones de precipitación y la falta de certidumbre en este aspecto (Aguilar et al., 2005; Magrin et al., 2014). Un estudio realizado en México, Guatemala, Honduras y Costa Rica en 2007, encontró cierta preocupación de los productores por el cambio en los patrones climáticos, con percepciones diferenciadas por regiones, a excepción de la menor fiabilidad de los patrones de lluvia respecto a décadas pasadas, la cual fue generalizada (Eakin et al., 2014). En un estudio realizado con productores de café en Nicaragua entre 2013 y 2014, Zuluaga et al. (2015) encontraron resultados muy similares a los encontrados en este estudio para Costa Rica; en el caso de Nicaragua, el $95 \%$ de los caficultores encuestados percibieron cambios en el clima en el último decenio, consenso en la tendencia de calentamiento, y porcentajes elevados (en torno al $50 \%$ ) de caficultores reportaron cambios en la frecuencia o estacionalidad de las lluvias, y en la mayor intensidad de los eventos hidrometeorológicos extremos (sequía e inundación).

La combinación entre el incremento de temperatura, el cambio de los patrones de precipitación y la mayor incidencia de eventos hidrometeorológicos extremos, está afectando los cultivos de café en las zonas de estudio. En línea con estas percepciones, se ha encontrado que el incremento de temperatura afectó de forma significativa la producción de café (Gay et al., 2006), mientras que la variación de la precipitación puede afectar la floración, el tejido foliar y el ciclo de vida de plagas y enfermedades, entre otros aspectos (CEPAL y CAC/SICA, 2014). Caficultores del sur de México reportaron pérdida de frutos y reducción de follaje en la planta, incremento de plagas y enfermedades a consecuencia de lluvias intensas y exceso de humedad, reducción de floración y frutos por altas temperaturas o exceso de lluvias, y secado de frutos por exceso de calor (Frank et al., 2011), daños similares a los reportados en este estudio.

A pesar de que la mayoría de los productores han experimentado los impactos del cambio climático, solamente una parte de ellos estaba implementando acciones para adaptarse. De aquellos caficultores que percibieron cambios en el clima, el $45 \%$ de Turrialba y el $78 \%$ de Los Santos habían realizado cambios de manejo en la finca en los últimos diez años en respuesta al clima. Algunos productores solo trataban de adaptarse a los cambios de temperatura o de precipitación y algunos (19\% en Turrialba y $43 \%$ en Los Santos) realizaban acciones para protegerse de ambas alteraciones climáticas. La práctica preferida entre paisajes y tipo de cambios era la siembra de árboles en la finca. En Turrialba es frecuente encontrar cafetales con policultivos comerciales o con árboles de servicio (Moguel y Toledo, 1999). Mientras que, en Los Santos hay mayor variedad de especies de sombra y lo mas frecuente es encontrar policultivos comerciales (Moguel y Toledo, 1999). La intensificación por medio del incremento en la aplicación de agroquímicos (pesticidas, herbicidas, fungicidas y fertilizantes) era bastante frecuente en la muestra. Algunas prácticas, como el manejo y conservación de suelo y agua, eran más empleadas en respuesta a cambios de precipitación. Las prácticas de tipo tecnológico, como la introducción de sistemas de riego, el uso de variedades mejoradas, o el cambio de calendario agrícola, fueron poco frecuentes en general y se asociaron más a cambios en precipitación (ej. riego en Turrialba, cambio de calendario agrícola en Los Santos). Las prácticas de diversificación reportadas fueron muy escasas y se concentraban principalmente en Turrialba.

El uso de estrategias de adaptación al cambio climático en la agricultura es un tema recurrente en la literatura, sin embargo, no ha sido estudiado en profundidad en los sistemas cafetaleros de la región centroamericana (Dasgupta et al., 2014). A nivel global, la adaptación en la agricultura se ha realizado principalmente por medio de ajustes en las prácticas agrícolas convencionales, como cambios en los calendarios de siembra y cosecha o variación en la frecuencia y cantidad de riego o insumos agrícolas, y mediante la incorporación de prácticas de conservación (Dasgupta et al., 2014). A nivel regional, Eakin et al. (2014), sugieren que las prácticas de conservación de suelos y en menor medida los cambios en la variedad de café sembrada, se implementan en respuesta a factores climáticos, mientras que la diversificación de actividades económicas y de cultivos, así como los cambios de área 
sembrada, responden a otros factores como el impacto de las plagas y razones económicas. Esto podría explicar la baja adopción de prácticas tecnológicas en respuesta a cambios en el clima encontrados en Turrialba y en Los Santos. Determinados factores socioeconómicos (como tamaño del hogar, extensión de la finca o trabajar fuera del hogar, entre otros) determinan la preferencia de las prácticas de adaptación utilizadas por los hogares (Zuluaga et al., 2015). Además, algunos grupos de prácticas como trabajar en la finca, invertir en infraestructura y cambiar los cultivos son complementarios (es decir, las familias tienden a implementarlos de forma conjunta) y que, por el contrario, el uso de insumos sintéticos y las prácticas agroforestales y de conservación de suelos son sustitutivas, es decir, generalmente no se realizan de forma simultánea (Zuluaga et al., 2015). Esto explicaría por qué algunos productores redujeron el uso de insumos en sus fincas, probablemente optando por prácticas agroecológicas como las chapias manuales (que tienen un efecto en la reducción del uso de insumos), mientras que otros optaron por incrementar su uso.

Los pronósticos indican que la temperatura continuará incrementando en las próximas décadas, los eventos hidrometeorológicos extremos intensificándose y que no hay certeza en los cambios de los patrones de precipitación. Es crítico que los gobiernos, la cooperación internacional y los técnicos apoyen a los pequeños caficultores a adaptarse al cambio climático. En este aspecto, se considera que las prácticas agroforestales y agroecológicas son más adecuadas para la adaptación de los pequeños productores, porque reducen el impacto del cambio climático y los eventos climáticos extremos (Holt-Giménez, 2002; Altieri y Koohafkan, 2008; Altieri et al., 2015). En la mayoría de los casos, este tipo de prácticas no requiere material externo a la finca, como sí es el caso de las medidas tecnológicas, ni crean dependencia de productos sintéticos, como es el caso de las medidas de intensificación (Whelan y Newsom, 2014), y se aprovecha el conocimiento previo de los productores.

La Adaptación basada en Ecosistemas provee los beneficios de las prácticas agroecológicas y beneficios sociales y económicos a los productores (Vignola et al., 2015). En este estudio se observó que de los usuarios de las prácticas de Adaptación basada en Ecosistemas consideradas en este estudio, percibían los beneficios de tipo adaptativo y los co-beneficios que aportan las prácticas, por lo que, los productores podrían estar más anuentes a implementarlas si hubiese apoyo gubernamental para la adaptación. Promover este tipo de prácticas no solo beneficia a los productores, sino que tiene un impacto positivo a nivel de paisaje, incrementando la conectividad y la provisión de servicios ecosistémicos de los agroecosistemas a los ecosistemas naturales.

Para promover medidas de adaptación al cambio climático adecuadas y eficientes para los pequeños caficultores costarricenses, se requiere incrementar el apoyo institucional, político y financiero, así como fortalecer las capacidades técnicas en el uso de los servicios ecosistémicos como estrategia de adaptación al cambio climático (Pramova et al., 2012; Harvey et al., 2017; Donatti et al. 2018). Las iniciativas nacionales que fortalecen el capital social, la equidad política y el empoderamiento de los pequeños productores son necesarias para reducir la vulnerabilidad de estos (Burke y Lobell, 2010; Ruiz-Meza, 2015). Dado que la toma de decisiones para la adaptación se realiza a varios niveles y puede promoverse mediante iniciativas públicas o privadas (complementarias o no) (Dasgupta et al., 2014), el éxito de los procesos de adaptación depende en gran medida del involucramiento de los actores de todos los niveles en la planificación (Fischersworring et al., 2015). Existen oportunidades para apoyar la adaptación a nivel gubernamental por medio de las Contribuciones Nacionales Determinadas (NDCs), y los mecanismos internacionales de apoyo a la adaptación y al cumplimiento de las metas de desarrollo sostenible (Viguera et al., 2018). En este aspecto, la promoción de prácticas que contribuyen de forma simultánea a los objetivos de mitigación y adaptación, a la vez que proveen beneficios adicionales como conservación o seguridad alimentaria (Harvey et al., 2014) constituyen una oportunidad adicional para la adaptación de la caficultura en Costa Rica.

En el contexto actual, y bajo los pronósticos de cambio para la región, la adaptación del sector cafetalero costarricense debe constituir una prioridad a nivel nacional. Si bien algunos productores ya están implementando prácticas de adaptación en las fincas de café en respuesta al clima, se requiere más apoyo institucional para 
incrementar y coordinar dichos esfuerzos, especialmente orientados a la adaptación de los pequeños productores, a incrementar su resiliencia socioeconómica y ambiental, y a mejorar el bienestar de los ecosistemas.

\section{Conclusiones}

Los pequeños caficultores costarricenses están siendo afectados por algunos impactos del cambio climático sobre sus cultivos y sus medios de vida. Algunos productores han implementado ciertas prácticas de manejo en sus fincas para adaptarse, sin embargo, los esfuerzos actuales son insuficientes para cubrir sus necesidades de adaptación. Esto se debe en parte a una capacidad de innovación limitada, como se refleja en la baja implementación de prácticas tecnológicas.

Los resultados presentados sobre la percepción de cambios en el clima y los esfuerzos de adaptación sirven de base para orientar las políticas públicas, los programas de extensión y las iniciativas de desarrollo e implementación de tecnologías hacia la promoción de la adaptación y el incremento de la resiliencia climática, económica y social de las familias y los sistemas productivos de café de pequeña escala. El uso de algunas prácticas de Adaptación basada en Ecosistemas (AbE) está extendido entre los pequeños productores de las zonas de estudio; el incremento en el uso de estas prácticas en cafetales contribuirá al mejoramiento de los ecosistemas y a la provisión de servicios ecosistémicos para la población en general.

La situación actual de la caficultura en Costa Rica resalta la necesidad de priorizar medidas de adaptación y facilitar el acceso de los pequeños productores de café a estas medidas. En este aspecto, las prácticas de AbE están alineadas con las necesidades de los productores y las metas específicas del sector, ya que contribuyen a mejorar los medios de vida y a mitigar el cambio climático. Tanto esta como otras estrategias de adaptación, requieren asistencia técnica apropiada, financiamiento para la puesta en marcha (especialmente la renovación de cafetales

y las mejoras tecnológicas), coordinación entre grupos de productores, prevención y diseño de estrategias de respuesta específica para distintos eventos hidrometeorológicos. Integrar estos aspectos en las políticas públicas y la planificación facilitarán la adaptación de los pequeños caficultores costarricenses.

\section{Agradecimientos}

Los autores agradecen el esfuerzo de Lucía Contreras, Marie Cadudal y Ahías Steller en la preparación de las bases de datos, reestructuración y revisión de los cuadros y figuras del documento, al equipo que participó en la recolección de datos y a los productores que compartieron sus conocimientos y percepciones con nosotros.

\section{Literatura citada}

Aguilar, E., T.C. Peterson, P. Ramírez, R. Frutos, J.A. Retana, M. Solera, J. Soley, I. González-García, R.M. Araujo, A. RosaSantos, V.E. Valle, M. Brunet, L. Aguilar, L. Álvarez, M. Bautista, C. Castañón, L. Herrera, E. Ruano, J.J. Sinay, E. Sánchez, G.I. Hernández-Oviedo, F. Obed, J.E. Salgado, J.L. Vásquez, M. Baca, M. Gutiérrez, C. Centella, J. Espinosa, D. Martínez, B. Olmedo, C.E. Ojeda-Espinoza, R. Núñez, M. Haylock, H. Benavides, and R. Mayorga. 2005. Changes in precipitation and temperature extremes in Central America and northern South America, 1961-2003. J. Geophys. Res. 110:D23107. doi:10.1029/2005JD006119

Alpizar, F., C.A. Harvey, M. Saborío-Rodríguez, B. Viguera, M.R. Martínez-Rodríguez, and R. Vignola. 2019. Household survey of climate change perception and adaptation strategies of smallholder coffee and basic grain farmers in Central America 20042014. UK Data Service, Colchester, Essex, GBR. http://reshare.ukdataservice.ac.uk/853252/ (consultado 30 mar. 2019). 
Alpízar, M., M. Chacón, K. López, C. Medaglia, E. Mora, L. Moreira, y S. Segura. 2015. Estadísticas de comercio exterior de Costa Rica 2014. PROCOMER, San José, CRC. http://www.procomer.com/uploads/downloads/anuario-estadistico-2014. pdf (consultado 9 ago. 2017).

Altieri, M.A., and P. Koohafkan. 2008. Enduring farms: climate change, smallholders and traditional farming communities. Third World Network, Penang, MAS.

Altieri, M.A., C.I. Nicholls, A. Henao, and M.A. Lana. 2015. Agroecology and the design of climate change-resilient farming systems. Agron. Sust. Dev. 35:869-890. doi:10.1007/s13593-015-0285-2

Bacon, C.M., W.A. Sundstrom, I.T. Stewart, and D. Beezer. 2017. Vulnerability to cumulative hazards: coping with the coffee leaf rust outbreak, drought, and food insecurity in Nicaragua. World Dev. 93:136-152. doi:10.1016/j.worlddev.2016.12.025

Bunn, C., P. Läderach, O. Ovalle-Rivera, and D. Kirschke. 2015. A bitter cup: climate change profile of global production of Arabica and Robusta coffee. Climatic Change 129:89-101. doi:10.1007/s10584-014-1306-x

Buppert, T., and A. McKeehan. 2013. Guidelines for applying free, prior and informed consent: a manual for conservation international. Conservation International, Arlington, VA, USA.

Burke, M., and D. Lobell. 2010. Food security and adaptation to climate change: What do we know? In: D. Lovell, and M. Burke, editors, Climate change and food security. Springer, Berlin, GER. p. 133-153.

Burnham, M., and Z. Ma. 2015. Linking smallholder farmer climate change adaptation decisions to development. Climate Develop. 8:289-311 doi:10.1080/17565529.2015.1067180

CBD (Secretariat of the Convention on Biological Diversity). 2009. Connecting biodiversity and climate change mitigation and adaptation: Key messages from the report of the second Ad Hoc technical expert group on biodiversity and climate change. CBD, Montreal, CAN. https://www.cbd.int/doc/publications/ahteg-brochure-en.pdf (accessed Aug. 9, 2017).

CEPAL (Comisión Económica para América Latina y el Caribe), y CAC/SICA (Consejo Agropecuario Centroamericano/ Sistema de la Integración Centroamericano). 2014. Impactos potenciales del cambio climático sobre el café en Centroamérica. CEPAL, MEX.

Conway, G. 2011. On being a smallholder. Presented at: Conference on New Directions for Smallholder Agriculture. IFAD, Rome, ITA, 24-25 Jan.

Cruz-Bello, G.M., H. Eakin, H. Morales, and J.F. Barrera. 2011. Linking multi-temporal analysis and community consultation to evaluate the response to the impact of hurricane stan in coffee areas of Chiapas, Mexico. Nat. Hazards 58:103-116. doi:10.1007/s11069-010-9652-0

Dasgupta, P., J.F. Morton, D. Dodman, B. Karapinar, F. Meza, M.G. Rivera-Ferre, A. Toure Sarr, and K.E. Vincent, 2014: Rural areas. In: C.B. Field et al., editors, Climate change 2014: impacts, adaptation, and vulnerability. Part A: global and sectoral aspects. Contribution of Working Group II to the Fifth Assessment Report of the Intergovernmental Panel on Climate Change. Cambridge University Press, Cambridge, GBR, and NY, USA. p. 613-657.

Donat, M.G., L.V. Alexander, H. Yang, I. Durre, R. Vose, R.J.H. Dunn, K.M. Willett, E. Aguilar, M. Brunet, J. Caesar, B. Hewitson, C. Jack, A.M.G. Klein Tank, A.C. Kruger, J.A. Marengo, T.C. Peterson, M. Renom, C. Oria Rojas, M. Rusticucci, J. Salinger, A. Sanhouri Elrayah, S.S. Sekele, A.K. Srivastava, B. Trewin, C. Villarroel, L.A. Vincent, P. Zhai, X. Zhang, and S. Kitching. 2013. Updated analyses of temperature and precipitation extreme indices since the beginning of the twentieth century: the HadEX2 dataset. J. Geophys. Res. Atmosph.118:2098-2118. doi:10.1002/jgrd.50150

Donatti, C.I., C.A. Harvey, M.R. Martinez-Rodriguez, R. Vignola, and C.M. Rodríguez. 2018. Vulnerability of smallholder farmers to climate change in Central America and Mexico: current knowledge and research gaps. Climate Dev. 5529:123. doi:10.1080/17565529.2018.1442796 
Eakin, H., L.A. Bojórquez-Tapia, R. Monterde-Diaz, E. Castellanos, and J. Haggar. 2011. Adaptive capacity and socialenvironmental change: theoretical and operational modeling of smallholder coffee systems response in mesoamerican pacific rim. Environ. Manag. 47:352-367. doi:10.1007/s00267-010-9603-2

Eakin, H., C.M. Tucker, E. Castellanos, R. Diaz-Porras, J.F. Barrera, and H. Morales. 2014. Adaptation in a multi-stressor environment: perceptions and responses to climatic and economic risks by coffee growers in Mesoamerica. Environ. Dev. Sustain. 16:123-139. doi:10.1007/s10668-013-9466-9

Fischersworring, B., G. Schmidt, K. Linne, P. Pringle, and P.S. Baker. 2015. Climate change adaptation in coffee production: A step-by-step guide to supporting coffee farmers in adapting to climate change. CABI. https://www.cabi.org/Uploads/ CABI/projects/Coffee\%20and\%20climate\%20change.pdf (accessed Sep.19, 2017)

Frank, E., H. Eakin, and D. López-Carr. 2011. Social identity, perception and motivation in adaptation to climate risk in the coffee sector of Chiapas, Mexico. Global Environ. Change 21:66-76. doi:10.1016/j.gloenvcha.2010.11.001

Gay, C., F. Estrada, C. Conde, H. Eakin, and L. Villers. 2006. Potential impacts of climate change on agriculture: A case of study of coffee production in Veracruz, Mexico. Climatic Change 79:259-288. doi:10.1007/s10584-006-9066-x

Hannah, L., C.I. Donatti, C.A. Harvey, E. Alfaro, D.A. Rodriguez, C. Bouroncle, E. Castellanos, F. Diaz, E. Fung, H.G. Hidalgo, P. Imbach, P. Läderach, J.P. Landrum, and A.L. Solano. 2017. Regional modeling of climate change impacts on smallholder agriculture and ecosystems in Central America. Climatic Change 141:29-45. doi:10.1007/s10584-016-1867

Harvey, C.A., M. Chacón, C.I. Donatti, E. Garen, L. Hannah, A. Andrade, L. Bede, D. Brown, A. Calle, J. Chará, C. Clement, E. Gray, M.H. Hoang, P. Minang, A.M. Rodríguez, C. Seeberg-Elverfeldt, B. Semroc, S. Shames, S. Smukler, E. Somarriba, E. Torquebiau, J. van-Etten, and E. Wollenberg. 2014. Climate-smart landscapes: Opportunities and challenges for integrating adaptation and mitigation in tropical agriculture. Conserv. Lett. 7(2):77-90. doi:10.1111/conl.12066

Harvey, C.A., M.R. Martínez-Rodríguez, J.M. Cárdenas, J. Avelino, B. Rapidel, R. Vignola, C.I. Donatti, and S. VilchezMendoza. 2017. The use of ecosystem-based adaptation practices by smallholder farmers in Central America. Agric. Ecosyst. Environ. 246: 279-290 doi:10.1016/j.agee.2017.04.018

Holdridge, L.R. 1947. Determination of world plant formations from simple climatic data. Science 105:367-368. doi:10.1126/ science.105.2727.367

Holt-Giménez, E. 2002. Measuring farmers' agroecological resistance after hurricane Mitch in Nicaragua: A case study in participatory, sustainable land management impact monitoring. Agric. Ecosyst. Environ. 93:87-105. doi:10.1016/S01678809(02)00006-3

ICAFE (Instituto del Café de Costa Rica). 2016. Informe sobre la actividad cafetalera de Costa Rica. ICAFE, Heredia, CRC. http:// www.icafe.cr/wp-content/uploads/informacion_mercado/informes_actividad/anteriores/2016.pdf (consultado 3 ago. 2017).

ICAFE (Instituto del Café de Costa Rica). s.f. Regiones cafetaleras de Costa Rica: Tarrazú. ICAFE, Heredia, CRC. http://www. icafe.cr/nuestro-Cafe/regiones-Cafetaleras/tarrazu/ (consultado 8 mar. 2018).

Imbach, P., M. Beardsley, C. Bouroncle, C. Medellin, P. Läderach, H. Hidalgo, E. Alfaro, J. Van-Etten, R. Allan, D. Hemming, R. Stone, L. Hannah, and C.I. Donatti. 2017. Climate change, ecosystems and smallholder agriculture in Central America: an introduction to the special issue. Climatic Change 141:1-12. doi:10.1007/s10584-017-1920-5

INEC (Instituto Nacional de Estadística y Censos). 2007. Censo cafetalero: Turrialba y Coto Brus 2003, Valle Central y Valle Central Occidental 2004, y Pérez Zeledón, Tarrazú y Zona Norte 2006. Principales resultados. Instituto del Café de Costa Rica, San José, CRC. http://www.inec.go.cr/censos/censos-cafetalero (consultado 9 ago. 2017).

INDER (Instituto de Desarrollo Rural). 2016. Plan de desarrollo rural territorial (PDRT) 2016-2021 - Los Santos. INDER, CRC. https://www.inder.go.cr/territorios_inder/region_central/planes_desarollo/PDRT-Los-Santos.pdf (consultado 8 mar. 2018). 
IPCC. 2012. Managing the risks of extreme events and disasters to advance climate change adaptation. Cambridge University Press, Cambridge, GBR, and NY, USA.

Läderach, P., J. Haggar, C. Lau, A. Eitzinger, O. Ovalle, M. Baca, A. Jarvis, and M. Lundy. 2013. Mesoamerican coffee: building a climate change adaptation strategy. CIAT Policy Brief No. 2. CIAT, Cali, COL.

Lin, B.B., I. Perfecto, and J. Vandermeer. 2008. Synergies between agricultural intensification and climate change could create surprising vulnerabilities for crops. BioSci. 58:847-854. doi:10.1641/B580911

Magrin, G.O., J.A. Marengo, J.P. Boulanger, M.S. Buckeridge, E. Castellanos, G. Poveda, F.R. Scarano, and S. Vicuña. 2014. Central and South America. In: V.R Barros et al., editors, Climate change 2014: Impacts, adaptation, and vulnerability. Part B: Regional aspects. Contribution of working group II to the fifth assessment report of the Intergovernmental Panel on Climate Change. Cambrige University Press, GBR and NY, USA. p. 1499-1556.

MIDEPLAN (Ministerio de Planificación Nacional y Política Económica), y SICA (Sistema de Integración Centroamericana). 2016. Plan cantonal de desarrollo humano local (PCDHL) 2016-2026: Cantón de Turrialba. Docplayer, ESP. https:// docplayer.es/53037001-Plan-cantonal-de-desarrollo-humano-local-pcdhl.html (consultado 8 mar. 2018).

Moguel, P., and V.M. Toledo. 1999. Biodiversity conservation in traditional coffee systems of Mexico. Conserv. Biol. 13(1):1121. doi:10.1046/j.1523-1739.1999.97153.x

Nagayets, O. 2005. Small farms: current status and key trends information brief. Edeias na mesa, BRA. https://www. ideiasnamesa.unb.br/upload/bibliotecaIdeias/1412955435small_farms.pdf (accessed Mar. 8, 2018).

Noble, I.R., S. Huq, Y.A. Anokhin, J. Carmin, D. Goudou, F.P. Lansigan, B. Osman-Elasha, and A. Villamizar. 2014. Adaptation needs and options. assess. In: C.B. Field et al., editors, Climate change 2014: Impacts, adaptation, and vulnerability. Part A: global and sectoral aspects. Contribution of working group II to the Fifth assessment report of the Intergovernmental Panel on Climate Change. Cambridge University Press, GBR and NY, USA. p. 833-868.

Ovalle-Rivera, O., P. Läderach, C. Bunn, M. Obersteiner, and G. Schroth. 2015. Projected shifts in coffea arabica suitability among major global producing regions due to climate change. PLoS ONE 10(4):e0124155. doi:10.1371/journal. pone. 0124155

Paiva, P. 2000. Economic and social development in Latin America: The role of coffee. Presented at: World Coffee Conference. May 18. Hilton Park Lane, GBR. http://www.ico.org/event_pdfs/paiva.pdf (Accessed Aug., 2017).

Pramova, E., B. Locatelli, M. Brockhaus, and S. Fohlmeister. 2012. Ecosystem services in the national adaptation programmes of action. Climate Policy 12:393-409. doi:10.1080/14693062.2011.647848

Ruiz-Meza, L.E. 2015. Adaptive capacity of small-scale coffee farmers to climate change impacts in the Soconusco region of Chiapas, Mexico. Clim. Dev. 7:100-109. doi:10.1080/17565529.2014.900472

Schroth, G., P. Laderach, J. Dempewolf, S. Philpott, J. Haggar, H. Eakin, T. Castillejos, J. Garcia-Moreno, L. Soto-Pinto, R. Hernandez, A. Eitzinger, and J. Ramirez-Villegas. 2009. Towards a climate change adaptation strategy for coffee communities and ecosystems in the Sierra Madre de Chiapas, Mexico. Mitig. Adapt. Strateg. Glob. Change 14:605-625. doi:10.1007/s11027-009-9186-5

SEPSA (Secretaría Ejecutiva de Planificación Sectorial Agropecuaria). 2015. Boletín estadístico agropecuario N²5. Serie Cronológica 2011-2014. InfoAgro, San José, CRC. http://www.infoagro.go.cr/BEA/BEA25/descargas/BEA25.pdf (consultado 9 ago. 2017).

Tucker, C.M., H. Eakin, and E.J. Castellanos. 2010. Perceptions of risk and adaptation: coffee producers, market shocks, and extreme weather in Central America and Mexico. Global Environ. Change 20:23-32. doi:10.1016/j.gloenvcha.2009.07.006 
Vignola, R., C.A Harvey, P. Bautista-Solis, J. Avelino, B. Rapidel, C. Donatti, and R. Martinez. 2015. Ecosystem-based adaptation for smallholder farmers: Definitions, opportunities and constraints. Agric. Ecosyst. Environ. 211:126.132. doi:10.1016/j.agee.2015.05.013

Viguera, B., M. R. Martínez-Rodríguez, F. Alpízar, y C.A. Harvey. 2018. Adaptación basada en ecosistemas como una opción de adaptación de la agricultura al cambio climático en Centroamérica. CATIE, y Conservación Internacional (CI), Turrialba, CRC.

Whelan, T., and D. Newsom. 2014. Sustainable coffee farming: improving income and social conditions protecting water, soil and forests. Rainforest Alliance, NY, USA.

Zuluaga, V., R.A. Labarta, and P. Läderach. 2015. Climate change adaptation: The case of coffee sector in Nicaragua. Present at: 2015 Agricultural \& Applied Economics Association and Western Agricultural Economics Association Annual Meeting, San Francisco, CA, USA. July 26-28. 